\title{
Asistencia a videoconferencias y aprendizaje en modalidad online: descripción de estrategias en estudiantes de formación del profesorado
}

Mercedes Romero Rodrigo - Universitat de València

Ángel San Martin Alonso - Universitat de València

José Peirats Chacón - Universitat de València
D 0000-0002-9103-5668

iD $0000-0003-3565-4250$

(D) $0000-0002-6580-2712$

Recepción: 18.12.2021 | Aceptado: 21.12.2021

Correspondencia a través de ORCID: Mercedes Romero Rodrigo

iD $0000-0002-9103-5668$

Citar: Romero, M, San Martín, A y Peirats, P (2021). Asistencia a videoconferencias y aprendizaje en modalidad online: descripción de estrategias en estudiantes de formación del profesorado. REIDOCREA, 10(41), 1-10.

Área o categoría del conocimiento: Multidisciplinar

Resumen: El estudio acerca de las estrategias empleadas por los estudiantes universitarios viene siendo una temática de interés para los investigadores cuya preocupación tiene que ver con el aprendizaje. Tras consultar las últimas aportaciones en el campo, queda corroborado que mayormente se han centrado en modalidades presenciales. Objetivo: Esclarecer el perfil estratégico de un grupo de estudiantes en modalidad no presencial, así como determinar la existencia o no de diferencias significativas en función del acceso a las videoconferencias. Método: Investigación cuantitativa cuyo instrumento de recogida de información fue el cuestionario de estrategias de aprendizaje CEVEAPEU (Gargallo et al., 2009). Resultados: Por un lado, se determinó la existencia de diferencias significativas en el empleo de estrategias, estableciéndose tres perfiles claramente diferenciados: alto, medio y bajo. Por otro, no se apreciaron diferencias significativas en función del grado de acceso a las sesiones en directo salvo en cuatro estrategias: atribuciones internas, control de la ansiedad, planificación, habilidades sociales y aprendizaje entre compañeros.

Palabra clave: aprendizaje en línea | estrategias de aprendizaje

Videoconference attendance and online learning: description of strategies in teacher education students

Abstract: The study of university students learning strategies has been a topic of interest for researchers whose concern has to do with learning. After consulting the latest contributions, it is corroborated that they have been mostly focused on face-to-face modalities. Objective: To clarify the strategic profile of a group of students in non-f of face-to-face modality as well as to determine the existence or not of significant differences depending on the access to videoconferences. Method: Quantitative research using the CEVEAPEU learning strategies questionnaire (Gargallo et al., 2009). Results: On the one hand, the existence of significant differences in the use of strategies was determined, establishing three clearly differentiated profiles: high, medium and low. On the other hand, no significant differences were found according to the degree of access to live sessions except in four strategies: internal attributions, anxiety control, planning, social skills and peer learning.

Keyword: e-learning | learning strategies

\section{Introducción}

Desde hace años en nuestro país, la investigación en torno a las estrategias de aprendizaje desplegadas por los estudiantes (ya fueran estos de secundaria o universidad) es una realidad. Basta con acudir a diversas bases de datos y realizar una búsqueda sobre la temática para corroborar la cantidad de literatura existente. Otra muestra de ello es la adaptación de diversos instrumentos de medida al contexto español, principalmente en los años 90 , debido a las diferencias referidas al leguaje y/o de tipo cultural. Años después se procedió, ya no tanto a realizar ajustes, sino a desarrollar instrumentos propios: 


\begin{tabular}{|c|c|c|c|}
\hline \multicolumn{4}{|c|}{ Tabla 1. Estrategias de aprendizaje e instrumentos de medida } \\
\hline Etapa & Autor & Acrónimo & Nombre \\
\hline \multirow{4}{*}{$\begin{array}{l}\text { Instrumentos } \\
\text { seminales }\end{array}$} & Schmeck et al. (1977) & ILP & . Inventory of Learning Processes \\
\hline & Weinstein et al. (1987) & LASSI & $\begin{array}{l}\text { Learning and Study Strategies } \\
\text { Inventory }\end{array}$ \\
\hline & Entwistle \& Ramsden (1982) & ASI & - Approaches to Studying Inventory \\
\hline & Pintrich et al. (1991) & MLSLQ & $\begin{array}{l}\text { Motivation Strategies for Learning } \\
\text { Questionnaire }\end{array}$ \\
\hline \multirow{5}{*}{$\begin{array}{l}\text { Instrumentos } \\
\text { desarrollados en } \\
\text { el contexto } \\
\text { español }\end{array}$} & Román y Gallego (1994) & ACRA & - Escalas de Estrategias de Aprendizaje \\
\hline & Roces et al. (1995) & CEAM & $\begin{array}{l}\text { Cuestionario de Estrategias de } \\
\text { Aprendizaje y Motivación }\end{array}$ \\
\hline & Alonso et al. (1994) & CHAEA & $\begin{array}{l}\text { Cuestionario de Honey-Alonso de } \\
\text { Estilos de Aprendizaje }\end{array}$ \\
\hline & Ayala et al. (2004) & CEAM II & $\begin{array}{l}\text { Cuestionario de Estrategias de } \\
\text { Aprendizaje y Motivación }\end{array}$ \\
\hline & Gargallo et al. (2009) & CEVEAPEU & $\begin{array}{l}\text { Cuestionario de Evaluación de las } \\
\text { Estrategias de Aprendizaje de los } \\
\text { Estudiantes Universitarios }\end{array}$ \\
\hline
\end{tabular}

Este hecho repercutió en el incremento de investigaciones en las siguientes décadas como mencionábamos anteriormente. Entre los hallazgos de las investigaciones más recientes sobre el tema que nos ocupa, destacan las mejoras significativas en estrategias de habilidades de interacción social y aprendizaje entre compañeros tras implementar simulaciones (García, 2016); la existencia de diferencias entre el estudiantado según la vía de acceso a la universidad (Tortajada y Chust, 2018); o el mejor dominio de estrategias afectivas, de apoyo y control frente a las relacionadas con el procesamiento de la información (Chiner et al., 2020). A estos descubrimientos cabe añadir líneas de investigación precedentes que quedaron constatadas, como la existencia de diferencias en el uso de estrategias y variables como el sexo de los estudiantes (Juárez et al., 2012), rendimiento académico (Gargallo y Suárez, 2014; Jiménez, 2015), motivación (Gargallo et al., 2012) o ansiedad (Furlan et al., 2009; García, 2011).

Partiendo de los antecedentes expuestos y teniendo en cuenta que todos ellos focalizaron sus investigaciones en estudiantes universitarios en modalidad presencial, situamos el problema de investigación en el desconocimiento en torno al uso de estrategias de aprendizaje en modalidades no presenciales.

\section{Hipótesis}

En base a lo expuesto en el apartado anterior, la presente investigación tuvo por objeto identificar los perfiles estratégicos de un grupo de estudiantes del Máster de Secundaria en modalidad no presencial. En lo que respecta a las hipótesis del presente trabajo, por una parte se pretendió demostrar la existencia de diferencias significativas en cuanto al uso de estrategias afectivas, de apoyo y control así como aquellas relacionas con el procesamiento de la información. Dichos resultados responderían a tres grupos diferenciados de alumnos en función del dominio de las estrategias indicadas, lo cual confirmaría la existencia de un grupo con un perfil de dominio alto, otro medio y un último con un nivel bajo o deficiente.

En segundo lugar, como objetivo adicional a esta investigación, se examinaron las diferencias en el uso de estrategias por parte de los estudiantes según el acceso a las sesiones de videoconferencia al considerarse una cuestión poco explorada en investigaciones anteriores y siendo un asunto no menor dada la naturaleza de la 
modalidad del Máster. La hipótesis a contrastar, por lo tanto, es la existencia de diferencias significativas en función de grado de acceso a las sesiones en directo, manifestando una mejor competencia estratégica en aquellos estudiantes cuya asistencia es mayor.

\section{Método}

\section{Participantes}

La muestra inicial estuvo formada por un total de 758 estudiantes de los cuales fueron descartados 52 al no participar en la contestación del cuestionario. Cabe señalar que este fue administrado en el marco de la asignatura de Innovación docente e iniciación a la investigación educativa como parte de una actividad de evaluación continua. Por tanto, aquellos estudiantes que no la realizaron quedaron excluidos de la investigación.

La muestra final la conformaron 706 personas, siendo 414 mujeres y 292 hombres, con un rango de edad que osciló entre los 22 a 47 años (376 contaban con 29 años o una edad inferior, 260 tenían entre 30 y 39 años mientras que los últimos 70 participantes tenían 40 años o más). Asimismo, los estudiantes indicaron su situación laboral (variable que tras los análisis resultó no ser significa en relación al uso de estrategias, razón por la cual no ha sido tenida en cuenta en el presente trabajo) así como el nivel de acceso a las sesiones de videoconferencia en directo.

\section{Instrumento}

Como instrumento de recogida de información se administró el Cuestionario de Evaluación de las Estrategias de Aprendizaje de los Estudiantes Universitarios (CEVEAPEU) de Gargallo et al. (2009). Se optó por este instrumento por varias razones: en primer lugar por estar destinado a estudiantes universitarios y elaborado teniendo en cuenta el contexto español; en segundo lugar, por contar con una escala específica que evalúa las estrategias de búsqueda, recogida y selección de la información (aspecto que no es tenido en cuenta en instrumentos similares), y en último lugar, por integrar tanto escalas motivacionales como una mejor definición de ítems relacionados con estrategias afectivas y metacognitivas. En total, está formado por 88 ítems a valorar mediante escala Likert donde 1=muy en desacuerdo; 2=en desacuerdo; $3=$ indeciso; 4= de acuerdo; $5=$ muy de acuerdo.

La primera escala de estrategias afectivas, de apoyo y control (o automanejo) cuenta con una consistencia interna de 0.819 ( $\alpha$ de Cronbach) y se organiza en: (a) estrategias motivacionales (motivación intrínseca -MOTIN-; motivación extrínseca -MOTEXT-; valor de la tarea -VALTAR-; atribuciones internas -ATRINT-; atribuciones externas -ATREXT; autoeficacia y expectativas -AUTOEFIC-; concepción de la inteligencia como modificable -CONINT-); (b) componentes afectivos (estado físico y anímico -ESTFIS- y ansiedad -CONTANS-); (c) metacognitivas (Conocimiento de objetivos y criterios de evaluación -CONOBJ-; planificación -PLANIF-; autoevaluación -AUTOEV-; control, autorregulación -CONTAUTOR-); (d) control del contexto, interacción social y manejo de recursos (control del contexto -CRTLCTX- y habilidades de interacción social y aprendizaje con compañeros -HABSOC-). La segunda, estrategias relacionadas con el procesamiento de la información, con una consistencia interna de 0.864 ( $\alpha$ de Cronbach) y está conformada por: (e) búsqueda y selección e información (conocimiento de fuentes y búsqueda de información -CONFUEN- y selección de información -SELINF-); (f) procesamiento y uso de la información (adquisición de la información -ADQINF-; elaboración -ELABINF-; organización -ORGINF-; personalización y creatividad, pensamiento crítico -PERCRE-; Almacenamiento, memorización. uso de recursos 
mnemotécnicos -ALMEN-; transferencia; uso de la información -TRANSF-; manejo de recursos para usar la información adquirida -MANREC-; almacenamiento simple, repetición -ALMSR-).

\section{Procedimiento y análisis estadísticos}

El cuestionario fue habilitado en el segundo cuatrimestre, momento en el que los estudiantes cursaron la asignatura anteriormente reseñada. El medio de acceso fue el propio LMS (Learning Management Sytem) o campus virtual, a través del cual se puso a disposición del estudiantado el enlace al Google Forms. Dicho formulario contenía tanto los 88 ítems como las preguntas previas de identificación. Manifestar que esta apuesta sobre el procedimiento de administración está motivada por investigaciones como la de Torrado (2004) y Díaz (2012) que ya apuntaron en su momento como ventajas, por ejemplo: la sensación de anonimato, lo cual potenciaría mayor sinceridad en las respuestas; o la reducción del sesgo del entrevistador (ya que éstos no son necesarios). En cuanto al proceso de análisis de datos se hizo uso del programa SPSS v24. A continuación, se detallan tanto los resultados obtenidos como los diversos análisis realizados en función de la hipótesis a contrastar.

\section{Resultados}

\section{Estrategias afectivas, de apoyo, control del contexto y procesamiento de la información: perfiles (grupos) en función del dominio de estrategias de aprendizaje}

Con la finalidad de obtener los resultados que mostrasen la existencia o no de diferencias significativas entre el uso de estrategias y los grupos (perfiles) se procedió a realizar un análisis de conglomerado de k-medias. En primer lugar, se acometió la realización de diversos sondeos, examinándose modelos de tres a seis conglomerados. En base a los criterios de parsimonia y congruencia de dichas opciones se verificó que el análisis de tres agrupamientos era el idóneo al presentar la mejor segmentación (el resto de las soluciones, en alguno de los casos, presentaba un único sujeto en uno de los grupos). Como resultado, se obtuvieron tres grupos diferenciados: el primero y que presentó un domino alto, formado por 248 estudiantes $(35,13 \%)$; el segundo y más numeroso, representando la mitad de la muestra $(52,69 \%, 372$ sujetos) con un dominio medio; y el tercero con un perfil más bajo y cuyo peso sobre el total representó el 12,18\% con 86 casos. Por consiguiente, quedó corroborado que la solución de tres agrupamientos es la más adecuada al obtenerse los resultados más consistentes y sólidos al igual de no resultar ni saturados o, por el contrario, dispersos.

Siguiendo con lo anterior y con la finalidad de comprobar la existencia o no de diferencias significativas entre los grupos se acometió un análisis multivariante de la varianza (MANOVA) a través del modelo lineal general, donde las estrategias de aprendizaje actuaron como variantes dependientes y grupos como independiente. A su vez, se elaboraron análisis descriptivos, estimación del tamaño del efecto, pruebas de homogeneidad de las varianzas, así como los efectos a nivel univariado (ANOVA). Según los resultados de $M$ de Box y su interpretación queda confirmado que no se cumple el supuesto de homogeneidad de las varianzas entre los grupos $(p \leq 0,000)$ lo cual nos indica a ser reservados a la hora de estimar los análisis de $\mathrm{F}$ en torno a la región de aceptación/rechazo. Con respecto al perfil multivariado queda confirmada la existencia de diferencias significativas según Lambda Wilks $\left(F_{50,1358}=35,599 ; p \leq 0,000\right)$ con un tamaño grande del efecto $\left(\eta^{2}\right.$ parcial $\left.=0,567\right)$ según Cohen $(1977)$. Para finalizar, indicar que las diferencias a nivel univariado fueron en todos los casos significativas, con un efecto muy importante tal y como puede ser verificado en la tabla siguiente. 


\begin{tabular}{|c|c|c|c|c|c|c|}
\hline Estrategias & Perfiles & Media & Desviación típica & $\mathrm{F}$ & Sig. & $\eta^{2}$ parcial \\
\hline \multirow{3}{*}{ MOTIN } & Alto & 4,5847 & 0,51692 & & & \\
\hline & Medio & 4,2285 & 0,45662 & 37761,582 &, 000 & ,982 \\
\hline & Bajo & 3,9767 & 0,51587 & & & \\
\hline \multirow{3}{*}{ MOTEXT } & Alto & 1,8044 & 0,72761 & & & \\
\hline & Medio & 1,8696 & 0,70601 & 3241,224 & ,000 & ,822 \\
\hline & Bajo & 2,0174 & 0,88931 & & & \\
\hline \multirow{3}{*}{ VALTAR } & Alto & 4,5907 & 0,38943 & & & \\
\hline & Medio & 4,2332 & 0,44421 & 45748,212 & ,000 & ,985 \\
\hline & Bajo & 3,8895 & 0,53907 & & & \\
\hline \multirow{3}{*}{ ATRINT } & Alto & 4,3575 & 0,4621 & & & \\
\hline & Medio & 4,129 & 0,4638 & 38802,076 &, 000 & ,982 \\
\hline & Bajo & 3,9535 & 0,48819 & & & \\
\hline \multirow{3}{*}{ ATREXT } & Alto & 2,3508 & 0,7365 & & & \\
\hline & Medio & 2,4167 & 0,7098 & 5638,419 & ,000 & ,889 \\
\hline & Bajo & 2,5174 & 0,68362 & & & \\
\hline \multirow{3}{*}{ AUTOEFIC } & Alto & 4,5363 & 0,4422 & & & \\
\hline & Medio & 4,1727 & 0,41074 & 46507 & ,000 & ,985 \\
\hline & Bajo & 3,9738 & 0,50443 & & & \\
\hline \multirow{3}{*}{ CONINT } & Alto & 3,0948 & 0,40505 & & & \\
\hline & Medio & 3,0228 & 0,37278 & 30595,013 &, 000 & ,978 \\
\hline & Bajo & 3,0756 & 0,40233 & & & \\
\hline \multirow{3}{*}{ ESTFIS } & Alto & 4,002 & 0,62017 & & & \\
\hline & Medio & 3,8152 & 0,53353 & 18660,392 & ,000 & ,964 \\
\hline & Bajo & 3,3169 & 0,79652 & & & \\
\hline & Alto & 2,9143 & 0,54221 & & & \\
\hline CONTANS & Medio & 2,918 & 0,51494 & 15352,76 &, 000 & ,956 \\
\hline & Bajo & 2,9477 & 0,50164 & & & \\
\hline & Alto & 4,3105 & 0,58349 & & & \\
\hline CONOBJ & Medio & 3,8925 & 0,56801 & 20841,527 & ,000 & ,967 \\
\hline & Bajo & 3,5116 & 0,75528 & & & \\
\hline & Alto & 3,5353 & 0,4594 & & & \\
\hline PLANIF & Medio & 3,2876 & 0,47469 & 23377,924 & ,000 & ,971 \\
\hline & Bajo & 3,0262 & 0,52304 & & & \\
\hline & Alto & 4,2473 & 0,42392 & & & \\
\hline AUTOEV & Medio & 3,9731 & 0,37661 & 44600,435 &, 000 & ,984 \\
\hline & Bajo & 3,624 & 0,52592 & & & \\
\hline & Alto & 4,3112 & 0,38333 & & & \\
\hline CONTAU-TOR & Medio & 3,8759 & 0,32592 & 52977,323 &, 000 & ,987 \\
\hline & Bajo & 3,5097 & 0,52291 & & & \\
\hline & Alto & 4,4446 & 0,47102 & & & \\
\hline CRTLCTX & Medio & 3,9032 & 0,50307 & 28299,463 &, 000 &, 976 \\
\hline & Bajo & 3,3895 & 0,66709 & & & \\
\hline & Alto & 3,9294 & 0,82148 & & & \\
\hline HABSOC & Medio & 3,6062 & 0,78451 & 9999,44 & ,000 & ,934 \\
\hline & Bajo & 3,4651 & 0,89884 & & & \\
\hline & Alto & 4,2107 & 0,51664 & & & \\
\hline CONFUEN & Medio & 3,6774 & 0,48575 & 24505,885 &, 000 & ,972 \\
\hline & Bajo & 2,9564 & 0,59803 & & & \\
\hline & Alto & 3,88 & 0,39026 & & & \\
\hline SELINF & Medio & 3,6163 & 0,28035 & 52409,514 &, 000 & ,987 \\
\hline & Bajo & 3,407 & 0,48666 & & & \\
\hline & Alto & 4,2876 & 0,52612 & & & \\
\hline ADQINF & Medio & 3,4991 & 0,53704 & 20529,334 &, 000 & ,967 \\
\hline & Bajo & 2,8372 & 0,64422 & & & \\
\hline & Alto & 4,6482 & 0,3696 & & & \\
\hline ELABINF & Medio & 4,1284 & 0,39001 & 55087,083 &, 000 & ,987 \\
\hline & Bajo & 3,7006 & 0,46149 & & & \\
\hline & Alto & 4,4242 & 0,55563 & & & \\
\hline ORGINF & Medio & 3,7597 & 0,64527 & 16926,227 & ,000 &, 96 \\
\hline & Bajo & 3,0581 & 0,80695 & & & \\
\hline & Alto & 4,3532 & 0,4518 & & & \\
\hline PERCRE & Medio & 3,8634 & 0,43913 & 36294,232 & ,000 & ,981 \\
\hline & Bajo & 3,5651 & 0,5377 & & & \\
\hline & Alto & 4,2446 & 0,69392 & & & \\
\hline ALMEN & Medio & 3,4624 & 0,76855 & 11032,038 &, 000 &, 94 \\
\hline & Bajo & 3,0969 & 0,88984 & & & \\
\hline & Alto & 1,5423 & 0,6979 & & & \\
\hline ALMSR & Medio & 1,8548 & 0,65909 & 3875,583 &, 000 &, 846 \\
\hline & Bajo & 2,4593 & 0,82325 & & & \\
\hline & Alto & 4,5874 & 0,40296 & & & \\
\hline TRANSF & Medio & 4,0717 & 0,41418 & 45522,26 &, 000 &, 985 \\
\hline & Bajo & 3,8488 & 0,57316 & & & \\
\hline & Alto & 4,3246 & 0,60236 & & & \\
\hline MANREC & Medio & 3,8051 & 0,54974 & 19315,081 &, 000 &, 965 \\
\hline & Bajo & 3,1453 & 0,77028 & & & \\
\hline
\end{tabular}


Con el propósito de explorar las relaciones que pudieran existir entre el uso de estrategias y el grado de acceso a las sesiones de videoconferencia, los alumnos fueron preguntados acerca del porcentaje de conectividad a las clases en directo. Las opciones de respuesta y los resultados obtenidos fueron los siguientes: (a) nunca - 1/4 parte de las sesiones (247 alumnos, lo que representó el 35\% de la muestra); (b) 1/4 - 2/4 (131; $18,6 \%$ ); (c) 2/4 - 3/4 (140; 19,8\%); (d) 3/4 - todas las sesiones (188; 26,6\%). Se partió de la premisa que, el estudiantado que se conectase a mayor número de sesiones presentaría unas mejores estrategias de aprendizaje.

Como se hiciera para contrastar la hipótesis anterior se acometió un análisis multivariante de la varianza (MANOVA), análisis descriptivos, estimación del efecto, pruebas de homogeneidad de las varianzas y efectos a nivel univariado (ANOVA) para cada estrategia de aprendizaje. Los resultados fueron los siguientes: $M$ de Box confirma que no se cumple el supuesto de homogeneidad de las varianzas entre grupos ( $p \leq$ $0,000)$, se confirma la existencia de diferencias significativas Lambda Wilks $(F 75,2027=2.521 p \leq 0,000)$. En cuanto a los resultados a nivel univariado (ANOVA):

Tabla 3. ANOVA, estadísticos descriptivos, F univariada y significación de las estrategias de aprendizaje según el grado de acceso a las clases en directo

\begin{tabular}{|c|c|c|c|c|c|c|}
\hline Estrategias & Acceso a videoconferencia en directo & Media & Desv. Típica & $\mathrm{F}$ & Sig. & $\eta^{2}$ parcial \\
\hline \multirow{4}{*}{ ATRINT } & $0 \%$ al $25 \%$ & 4,17 & 0,501 & \multirow{4}{*}{2,767} & \multirow{4}{*}{041} & \multirow{4}{*}{0,012} \\
\hline & $25 \%$ a $50 \%$ & 4,1959 & 0,46753 & & & \\
\hline & $50 \%$ al $75 \%$ & 4,1119 & 0,44991 & & & \\
\hline & $75 \%$ a $100 \%$ & 4,2624 & 0,49447 & & & \\
\hline \multirow{4}{*}{ CONTANS } & $0 \%$ al $25 \%$ & 2,8482 & 0,53031 & \multirow{4}{*}{3,405} & \multirow{4}{*}{017} & \multirow{4}{*}{0,014} \\
\hline & $25 \%$ a $50 \%$ & 2,9103 & 0,52903 & & & \\
\hline & $50 \%$ al $75 \%$ & 2,9411 & 0,52681 & & & \\
\hline & $75 \%$ a $100 \%$ & 3,0066 & 0,49356 & & & \\
\hline \multirow{4}{*}{ PLANIF } & $0 \%$ al $25 \%$ & 3,2176 & 0,52379 & \multirow{4}{*}{10,846} & \multirow{4}{*}{, 000} & \multirow{4}{*}{0,044} \\
\hline & $25 \%$ a $50 \%$ & 3,3282 & 0,52172 & & & \\
\hline & $50 \%$ al $75 \%$ & 3,3893 & 0,46387 & & & \\
\hline & $75 \%$ a $100 \%$ & 3,4827 & 0,44635 & & & \\
\hline \multirow{4}{*}{ HABSOC } & $0 \%$ al $25 \%$ & 3,3462 & 0,91024 & \multirow{4}{*}{34,136} & \multirow{4}{*}{, 000} & \multirow{4}{*}{0,127} \\
\hline & $25 \%$ a $50 \%$ & 3,6425 & 0,75874 & & & \\
\hline & $50 \%$ al $75 \%$ & 3,9083 & 0,64451 & & & \\
\hline & $75 \%$ a $100 \%$ & 4,0594 & 0,68068 & & & \\
\hline
\end{tabular}

Por tanto y a diferencia del apartado anterior, donde la hipótesis quedó totalmente corroborada, en este caso no se obtuvieron resultados significativos en todas las estrategias: solamente se dieron en atribuciones internas, control de la ansiedad, planificación, habilidades de interacción social y aprendizaje con compañeros. Adicionalmente indicar que el tamaño del efecto fue pequeño entre dichas estrategias con diferencias significativas y el acceso a las sesiones ya que $\eta^{2}$ parcial estuvo por debajo de 0,059 en todos los casos salvo en habilidades sociales, con un efecto mediano (Cohen, 1977). Por lo tanto, la hipótesis queda rechazada en gran medida.

\section{Discusión}

Antes de proceder a la discusión de resultados cabe aclarar un par de aspectos referidos a los resultados obtenidos, así como a su interpretación. Las puntuaciones medias oscilan entre 1 y 5 puntos dado que las preguntas formuladas debían ser respondidas haciendo uso de una escala tipo Likert. Por consiguiente, los resultados por encima de 4 puntos son indicadores de un buen dominio de la estrategia, por el contrario, aquellas 
que estén cercanas al 2 o resulten inferiores significarán todo lo contrario. Esto es así en todas las estrategias salvo en cuatro: motivación extrínseca, atribuciones externas, control de la ansiedad y almacenamiento simple. En estos casos se trata de estrategias invertidas, lo que implica que una mayor puntuación es indicador de un uso inadecuado, ya que está relacionado con conductas, estados, motivaciones, etc. que poco contribuyen al aprendizaje y/o lo dificultan. Por ejemplo, en el caso de la motivación extrínseca y atribuciones externas supone que el estudiantado responsabiliza de su situación académica a otros o bien actúa de una determinada manera para satisfacer a los demás (Bustos, 2016; Juárez et al., 2016; Tortajada y Chust, 2018).

De los resultados obtenidos se desprende que el grupo más numeroso de estudiantes presentan un perfil medio: obtienen puntuaciones adecuadas en estrategias de tipo motivacional y presentan un buen dominio de habilidades en la elaboración de información, así como en su transferencia. Si bien son estudiantes que no presentan problemas en las conocidas estrategias invertidas, las puntuaciones en estrategias relacionadas con el procesamiento de la información son mejorables. En comparación con el grupo que presentó un perfil estratégico alto, los cuales lograron puntuaciones por encima de 4,5 en diversas estrategias y las más bajas en lo que se refiere a las invertidas, el grupo medio debe perfeccionar cuestiones diversas. Primeramente convendría perfeccionar las estrategias de control del contexto (lugar de estudio, concentración y ambiente, aprovechamiento del tiempo), tan necesarias para obtener el máximo rendimiento (García, 2016) del mismo modo que ya fuera advertido en el estudio de Piovano et al. (2018), en el que los alumnos también presentaron deficiencias. Seguidamente, estrategias de interacción social (trabajo en equipo, resolución de dudas y ayuda entre iguales) ya que ello conlleva a que se cuente con recursos sociocognitivos que permitirán adecuarse a diversos contextos de aprendizaje (Jerónimo et al., 2019). Y por último manejo de recursos (organización para la preparación o respuesta a un examen) puesto que el nivel de preparación junto con factores de índole personal, son explicativos del fracaso académico (Chiner et al., 2019; Chiner et al., 2020).

Así mismo, por lo que respecta a las estrategias búsqueda, recogida y selección de la información, este grupo comparte con el de perfil bajo el mismo déficit. Cierto es que en parte de las estrategias de procesamiento y uso de la información obtienen buenas puntuaciones, pero dado que se trata de una escala con una fuerte relación entre sus estrategias (el dominio satisfactorio o deficiente impacta claramente en el resto) resulta aconsejable reforzarlas. Entre otras cosas, realizar gráficos, tablas, esquemas 0 resúmenes (estrategia de organización de la información) es un paso posterior a actividades como la prelectura, lectura comprensiva o toma de apuntes (estrategia de elaboración). Digamos que si ellas no se aplican o se hacen de una manera inadecuada el siguiente paso, como podría ser el uso de palabras clave o la mnemotecnia (estrategia de almacenamiento) presentará errores de base. Y así continuará sucediéndose en estadios posteriores como a la hora de aplicar lo aprendido (almacenamiento, repetición y trasferencia).

En último término y contrariamente a la premisa de partida, la asistencia a sesiones de videoconferencia en directo no resultó ser una variable indicadora de un mejor dominio de estrategias por parte del estudiantado. La razón que puede contribuir a explicar esta situación es que las estrategias, su conocimiento, uso y dominio es un proceso permanente y consciente orientado al aprendizaje (Gargallo et al., 2009; Melitón et al., 2018; Piovano et al., 2018). Ello implica que cada sujeto, a lo largo de su vida, aprende a cómo enfrentar situaciones diversas, a resolver problemas, a adaptarse a los objetivos de evaluación o metodología docente, entre otras. Y en este punto, la asistencia a clases en directo (más teniendo en cuenta la posibilidad de acceder a la grabación en caso de no conectarse en el horario establecido) no modificaría las estrategias a desplegar por los estudiantes, manteniendo un patrón de uso que ya ha sido adquirido. De un modo 
similar, la Tesis Doctoral de Bustos (2016), señaló que la falta de diferencias significativas en variables como el centro de estudios, sexo o edad, entre los colectivos analizados se debía a que los grupos manejaban patrones de pensamiento con rutas concretas para alcanzar sus objetivos. Esto se observa claramente en la segunda subescala: ninguna de las once estrategias relacionadas con la búsqueda, selección, así como procesamiento y uso de la información resultaron significativas, por lo que la asistencia a las videoconferencias no modificaba dicho camino.

En cambio, esta explicación no sería aplicable a las cuatro estrategias que si resultaron significativas. Los casos más evidentes son HABSOC y CONTANS. En el primero, la estrategia de habilidades sociales y aprendizaje entre compañeros es lógico esperar que se obtengan mejores puntuaciones conforme la asistencia crece. Téngase en cuenta que estudios como el de Vasquez y Gabalán (2017) y Chiner et al., (2020) han corroborado que existe relación entre la asistencia y el rendimiento, siendo a su vez una deducción generalmente aceptada por el estudiantado. Siguiendo en este orden de ideas, gracias a las sesiones en directo se pueden potenciar sinergias y el aprendizaje compartido entre los asistentes, cosa que no ocurre con el visionado de una grabación (Colomo et al., 2019; Gabarda et al., 2019). En el segundo, que la estrategia invertida de ansiedad obtenga peores resultados cuanto más se asiste a clase, sería comprensible teniendo en cuenta que los alumnos que presentan un menor dominio serían los que más se conectan: por ejemplo, para tener un mejor conocimiento del temario explicado por el docente y reducir así los nervios de cara al examen. Si bien no se han hallado estudios similares que compartan dicha explicación, no menos es cierto que los resultados referidos a la estrategia de control de la ansiedad suelen presentarse deficientes con cierta frecuencia (Chiner et al., 2020; Fernández et al., 2016; Gargalllo et al., 2014; Tortajada y Chust, 2018). Por ende, convenimos en señalar la necesidad de continuar indagando en este campo y en los motivos que puedan explicar este fenómeno.

\section{Conclusión}

Para finalizar, el presente trabajo ha indagado en el uso de estrategias de aprendizaje por parte de estudiantes universitarios del Máster en Educación Secundaria en una modalidad no presencial. Teniendo presente que se trata de futuros docentes, conviene subrayar que es deseable que presenten un buen dominio de estrategias, no solo por su trayectoria académica y éxito en los estudios (ámbito personal), sino porque en un espacio corto de tiempo podrían estar ante estudiantes que requieran de ayuda y orientación en este sentido (ámbito profesional).

Los resultados han puesto de manifiesto la existencia de tres grupos diferenciados si bien en dos de ellos coinciden en la necesidad de desarrollar y/o profundizar en estrategias relacionadas con la búsqueda y procesamiento de la información. En lo que concierne a la asistencia a las clases en directo, planteamiento diferente con respecto a la conocida como presencialidad híbrida (termino cada vez extendido como consecuencia del COVID-19) se ha constatado que no es indicador de diferencias en la puesta en marcha de unas estrategias u otras. Como nota discordante, la estrategia de habilidades de interacción social y aprendizaje con compañeros, en cuyo caso se dio una relación significativa. Esta circunstancia es indicador de que la videoconferencia como herramienta contribuye al desarrollo del aprendizaje entre iguales, lo cual implica que un buen uso de la misma permite la adquisición de competencias a la par de confirmarla como una apuesta coherente, más si cabe, teniendo presente los tiempos que corren. 
Como limitación y prospectiva de investigación del presente trabajo compartir la conveniencia de ampliar la muestra junto con la inclusión de otras titulaciones. Por un lado, se podrían obtener datos más significativos posibilitando la generalización de los mismos. Por otro, incorporar otras titulaciones ofrecería una imagen más compleja de los estudiantes universitarios, pudiendo explorarse las diferencias entre áreas de conocimiento y ofreciendo así recomendaciones de mejora específicas para cada grupo.

\section{Referencias}

Alonso, C., Gallego, D. y Honey, P. (1994). Los estilos de aprendizaje. Procedimientos de diagnóstico y mejora. Bilbao: Ediciones Mensajero.

Ayala, C.L, Martínez, R. y Yuste, C. (2004). Cuestionario de Estrategias de Aprendizaje y Motivación CEAM. Madrid: Instituto de Orientación Psicológica EOS.

Bustos, V. (2016). Estrategias de aprendizaje, actitudes emprendedoras, autoconcepto, esperanza, satisfacción vital e inclinación al autoempleo: un modelo explicativo en universitarios peruanos [Tesis Doctoral]. Valencia: Universitat de València.

Chiner, E., Cardona, M. C., Gómez, M., Villegas, Garcá, V. E. y Miralles, C. (2019). Análisis de las estrategias de aprendizaje de los estudiantes universitarios como indicadores del éxito académico. En R. Roig, J. M. Antolí, R. Díez y N. Pellín (coords). Memòries del Programa de Xarxes-I3CE de qualitat, innovació $i$ investigació en docència universitària, pp. 469-474.

Chiner, E., Gómez, M. y García, V. E. (2020). El uso de estrategias de aprendizaje como indicador del éxito académico en estudiantes universitarios. En R. Roig (coord) La docencia en la Enseñanza Superior: Nuevas aportaciones desde la investigación e innovación educativas, pp. 100-109.

Cohen, J. (1977). Statistical power analysis for behavioral sciences. New York: Academic Press.

Colomo, E., Gabarda, V., Cuevas, N. y Cívico, A. (2019). Las herramientas tecnológicas al servicio de la comunicación en las Prácticas de Magisterio: percepción de estudiantes y tutores sobre su utilidad. Revista Prácticum, 4(2), 24-41. https://doi.org/10.37042/practicum.2019.4.2.2

Díaz, V. (2012). Ventajas e inconvenientes de la encuesta por Internet. Papers: revista de sociologia, $\quad$ 97(1), 193-223. https://doi.org/10.5565/rev/papers/v97n1.71

Entwistle, N. J. y Ramsden, P. (1982). Understanding Student Learning. New York: Nichols Pub. Co.
Fernández, E., Ordoñez, T., Suria, R., Martínez, D. y Gras, L. (2016). PAT Ciencias: Programa de apoyo del CAE para detectar problemas de estrés, ansiedad $y / 0$ falta de autoestima en estudiantes de la Facultad de Ciencias. En R. Roig, J. E. Blasco, A. Lledó y N. Pellín (coords) Investigación e Innovación Educativa en Docencia Universitaria. Retos, Propuestas y Acciones, pp. 3091-3105.

Furlan, L. A., Sánchez, J., Heredia, D., Piemontesi, S. y Illbele, A. (2009). Estrategias de aprendizaje y ansiedad ante los exámenes en estudiantes universitarios. Pensamiento Psicológico, 5(12), 117-123.

Gabarda, V., Colomo, E. y Romero, M. (2019). Metodologías didácticas para el aprendizaje en línea. REIDOCREA, 8(2), 19-36.

García, A. (2011). Efectos del estrés percibido y las estrategias de aprendizaje cognitivas en el rendimiento académico de estudiantes universitarios noveles de ciencias de la salud. Málaga: Universidad de Málaga.

García, A. (2016). Aprender sin dañar. Motivación $y$ estrategias de aprendizaje de los alumnos del Grado de Enfermería de la UCAM que cursan simulación clínica. [Tesis Doctoral]. UCAM: Universidad Católica San Antonio.

Gargallo, B. y Suárez, J. (2014). Una aproximación al perfil de los estudiantes universitarios excelentes. Revista de docencia Universitaria, 12(2), 143-165.

Gargallo, B., Almerich, G., Suárez, J. y García, F. (2012). Estrategias de aprendizaje en estudiantes universitarios excelentes y medios. Su evolución a lo largo del primer año de carrera. Relieve, 18(2), 1-22.

Gargallo, B., Garfella, P. R., Sahuquillo, P., Verde, I. y Jiménez, M. A. (2015). Métodos centrados en el aprendizaje, estrategias y enfoques de aprendizaje en estudiantes universitarios. Revista de educación, 370, 229254.

Gargallo, B., Suárez, J., Pérez, C. (2009). El cuestionario CEVEAPEU. Un instrumento para la 
evaluación de las estrategias de aprendizaje de los estudiantes universitarios. Relieve, 15(2), 131. https://doi.org/10.7203/relieve.15.2.4156

Jerónimo, L. C., Yaniz-Álvarez, C. y Carcamo, C. (2020). Estrategias de aprendizaje de estudiantes colombianos de grado y posgrado. Magis: Revista Internacional de Investigación en Educación, 13(1) $1-20$. https://doi.org/10.11144/javeriana.m13.eaec

Jiménez, R. (2015). Análisis de los procesos de aprendizaje y el rendimento académico de los estudiantes universitarios y su relación con dimensiones personales y contextuales [Tesis Doctoral]. Valencia: Universitat de València.

Juárez, C. S., Rodríguez, G., Escoto, Mํㅡㄹ C. y Luna, E. (2016). Relación de los estilos y estrategias de aprendizaje con el rendimiento académico en estudiantes universitarios. Revista estilos de aprendizaje, 9(17), 268-288.

Juárez, C., Rodríguez, G. y Luna, E. (2012). El cuestionario de estilos de aprendizaje CHAEA y la escala de estrategias de aprendizaje ACRA como herramienta potencial para la tutoría académica. Revista de Estilos de Aprendizaje, 10(10), 148-171.

Melitón, P., Gallegos, S., Vilca, G. y López, M. (2018). Estrategias de aprendizaje en estudiantes universitarios de ciencias sociales: un estudio empírico en la escuela profesional de sociología de la UNAP. COMUNI@CCIÓN: Revista de Investigación en Comunicación y Desarrollo, 9(1), 35-47.

Pintrich, P., Smith, D., García, T. y McKeachie, W. (1991). A manual for the use of the Motivated Strategies for Learning Questionnaire (MSLQ). National Center for Research to Improve Postsecondary Teaching and Learning. Ann Arbor: University of Michigan.
Piovano, S., Roisen, E., Rodríguez, G. y Victorero, B. (2018). Estrategias de aprendizaje que utilizan los estudiantes de $1^{\circ}$ año de los departamentos de psicología y ciencias pedagógicas, administración y ciencias sociales y sistemas de una universidad privada. Revista Argentina de Educación Superior, (17), 98-114.

Roces, C., Tourón, J. y González, M.C. (1995). Validación preliminar del CEAM II (Cuestionario de Estrategias de Aprendizaje y Motivación II). Psicológica, 16(3), 347-366.

Román, J. M. y Gallego, S. (1994). ACRA. Escalas de estrategias de aprendizaje. Madrid: TEA.

Schmeck R., Ribich, F. y Ramanaiah, N. (1977). Development of a self-report inventory for assessing individual differences in learning processes. Applied Psychological Measurement, 41, 413-431.

Torrado, M. (2004). Estudios de encuesta. En R. Bisquerra (Coord.), Metodología de la investigación educativa (pp. 231-258). Madrid: La Muralla.

Tortajada, F. J. y Chust, P. (2018). Estrategias de aprendizaje en estudiantes universitarios de nuevo ingreso y su relación con la vía de acceso a la universidad. Congreso In-Red: Congreso Nacional de Innovación Educativa y de Docencia en Red. Universidad Politécnica de Valencia, 19 y 20 de julio de 2018, 814-825.

Vasquez, F. E. y Gabalán, J. (2017). Rendimiento académico universitario y asistencia a clases: Una visión. Revista de Educación, 41(2), 16-32. http://dx.doi.org/10.15517/revedu.v41i2.18477

Weinstein, C. E., Woolfolk, A., Palmer, D. y Schulte, A. C. (1987). Learning and study strategies inventory (LASSI). Clearwater, Florida: $\mathrm{H} \& \mathrm{H}$ Publishing Company. 